\section{Prediction of the cumulated dose for external beam irradiation of prostate cancer patients with 3D-CRT technique}

\begin{abstract}
Nowadays in radiotherapy, much effort is taken to minimize the irradiated volume and consequently minimize doses to healthy tissues. In our work, we tested the hypothesis that the mean dose distribution calculated from a few first fractions can serve as prediction of the cumulated dose distribution, representing the whole treatment. We made our tests for 25 prostate cancer patients treated with three orthogonal fields technique. We did a comparison of dose distribution calculated as a sum of dose distribution from each fraction with a dose distribution calculated with isocenter shifted for a mean setup error from a few first fractions. The cumulative dose distribution and predicted dose distributions are similar in terms of gamma (3 $\mathrm{mm} \mathrm{3 \% )}$ analysis, under condition that we know setup error from seven first fractions. We showed that the dose distribution calculated for the original plan with the isocenter shifted to the point, defined as the original isocenter corrected of the mean setup error estimated from the first seven fractions supports our hypothesis, i.e. can serve as a prediction for cumulative dose distribution.
\end{abstract}

Key words: 3D-CRT $\bullet$ cumulative dose $\bullet$ dose prediction $\bullet$ setup error $\bullet$ individualized margins

\section{Giżyńska}

Department of Medical Physics,

Maria Sklodowska-Curie Memorial Cancer Centre

and Institute of Oncology,

15B Wawelska Str., 02-034 Warsaw, Poland

and Biomedical Physics Division,

Institute of Experimental Physics,

Faculty of Physics,

University of Warsaw,

5 Pasteura Str., 02-093 Warsaw, Poland,

E-mail: marta.gizynska@fuw.edu.pl

D. Blatkiewicz, B. Czyżew, M. Gil-Ulkowska,

P. Kukołowicz

Department of Medical Physics,

Maria Sklodowska-Curie Memorial Cancer Centre

and Institute of Oncology,

15B Wawelska Str., 02-034 Warsaw, Poland

\section{Gałecki}

Biomedical Physics Division,

Institute of Experimental Physics,

Faculty of Physics,

University of Warsaw,

5 Pasteura Str., 02-093 Warsaw, Poland

Received: 1 August 2014

Accepted: 17 September 2015

\section{Introduction}

The purpose of treatment planning is to deliver a high uniform dose to the clinical target volume (CTV), while sparing the normal tissues. In order to achieve this goal, much effort is undertaken to minimize a margin added to CTV, while creating planning target volume (PTV) [1]. There are several approaches to define the margin added to CTV. Two of them, proposed by van Herk [2] and Stroom [3], are in common use. These models are population-based models. To minimize the margin, the individualized methods of creating the margin are searched [4-6]. The purpose of this work was to test the hypothesis that the predicted cumulated dose distribution calculated with the use of setup error measured in the few first fractions is a good estimate of the cumulated dose distribution, representing the whole treatment. The cumulative dose distribution is rather calculated as a sum of dose distributions calculated with isocenter shifted by a random error [2]. Our proposal leads to individualization of cumulative dose distribution. The work was performed for prostate patients because we had the full set of results of portal control for this group of patients. In the first step of our work, we decided to check our method for 3D cranial release technique (3D-CRT), which is easier to interpret. If our hypothesis is true, it would enable to individualize the CTV to PTV margin for each single patient. 


\section{Methods}

For 25 patients with prostate cancer treated in our clinic with three fields 3D conformal radiotherapy technique (3D-CRT) (anterior-posterior field and two lateral wedged fields) orthogonal portal images have been taken each day of 25 fractions (2.6 Gy each). Patients had empty rectum and full bladder (they drank 0.51 of water 30 min before adequate activity) during CT examination and irradiation as well. Knee-wedge support was used in order to provide better comfort and position reproducibility. CTV included prostate with seminal vesicles. PTV was created by adding following margins to the CTV: $7 \mathrm{~mm}$ in anterior-posterior and head-feet direction, $4 \mathrm{~mm}$ in left-right direction [7]. The setup errors, defined here as the difference between bones position with respect to the isocenter defined during planning on digital radiographs (DRR) and images taken before irradiation on the daily basis, were measured for each fraction. In order to do that, orthogonal DRRs were prepared in the treatment planning system. They were compared with orthogonal $\mathrm{kV}$ and MV images taken before irradiation on the daily basis. The positions of the bones relative to the isocenter position were compared. Simplifying the scenario, we assumed that the target volume is not moving independently from bones. It was shown [7] that one can find a vector between the prostate shift and bones. That is why we did this simplification and took into account only bony shift, which allowed us to use planning CT for recalculations. The actual dose distribution for each fraction was calculated with the isocenter corrected for setup errors along each of the three axes. The number of monitor units (MUs), quantity related to dose and machine output, for each field was preserved as in the original plan. In other words, radiation produced by medical accelerator (in its shape, energy, dose rate, and delivery time) was preserved [6]. All calculations were done in Eclipse Treatment Planning System v. 10.0 (Varian Medical Co., Palo Alto, USA) with analytical anisotropic algorithm (AAA) (v. 10.0.28). Next we have calculated the sum of dose distributions obtained for each single fraction (cumulated dose distribution $\left[\mathrm{CDD}_{\mathrm{all}}\right]$ ) and we have also calculated the dose distribution representing the mean setup error for $n$-th first fractions (predicted cumulated dose distribution $\left[\mathrm{PCDD}_{N}\right]-\mathrm{PCDD}_{2}, \ldots, \mathrm{PCDD}_{15}$, $\mathrm{PCDD}_{20}, \mathrm{PCDD}_{25}$ ). The $\mathrm{PCDD}_{N}$ was calculated using the original plan with the isocenter placed at the point whose coordinates were calculated as the mean shift calculated over first 2, 3, .., 15, 20, and 25 fractions.

Each $\mathrm{PCDD}_{N}$ was compared with $\mathrm{CDD}_{\text {all }}$ in terms of the orthogonal profiles calculated in isocenter plane, i.e., anterior-posterior (AP), left-right (LR), and head-feet (HF) profile. The gamma index [8] with $3 \mathrm{~mm}$ and $3 \%$ was used. Calculations were performed in Scilab-based homemade program. According to methodology used in radiation therapy (RT) plan verification, we assumed that dose distributions were equal if $95 \%$ of analyzed points had gamma index value not larger than 1 . Because the aim of the radiotherapy is to deliver the prescribe dose to the CTV, the planned dose distribution and the $\mathrm{PCDD}_{N}$ were also compared.

We used Kruskall-Wallis [9] and Kolmogorov-Smirnov [10] tests for comparison of CTV dose distribution (dose volume histograms [DVH]) between $\mathrm{CDD}_{\text {all }}$ and $\mathrm{PCDD}_{N}$. The null hypothesis was that this distributions do not differ. In 1984, Brahme [11] showed that effective dose delivered to the target is well estimated by the mean dose if the dose distribution in the PTV is highly uniform, which is the case in prostate patient plan. Based on this idea, we tested the null hypothesis that the dose distribution in CTV for $\mathrm{PCDD}_{N}$ is the same as for $\mathrm{CDD}_{\text {all }}$ in terms of the mean dose to the CTV. Wilcoxon test was used for test this hypothesis. Additionally, we also analyzed the changes of the minimum dose in the CTV as it is still reported in 3D-CRT radiotherapy practice.

\section{Results}

For AP profile, the maximum gamma index (i.e., the maximum value of gamma index calculated in method described previously) was 2.52 for all patients and all $\mathrm{PCDD}_{N}$. For 22 patients for the $\mathrm{PCDD}_{N>7}$ the gamma index was always lesser than 1.00. For LR profile, the maximum gamma index was 2.20. For 23 patients for the $\mathrm{PCDD}_{N>4}$ the gamma index was not larger than 1.00. For HF profile, the maximum gamma index was 1.78 . For 23 patients for the $\mathrm{PCDD}_{N>4}$ the maximum gamma index was not larger than 1.00. The number of patients with gamma index less than 1.00 in less than 95\% of points were: one patient for AP profile and $\mathrm{PCDD}_{6}$ to $\mathrm{PCDD}_{10}$, no patients for AP profile and $\mathrm{PCDD}_{11}$ to $\mathrm{PCDD}_{25}$, one patient for LR profile and $\mathrm{PCDD}_{7}$ to $\mathrm{PCDD}_{25}$, and one or no patients for $\mathrm{HF}$ profile and $\mathrm{PCDD}_{3}$ to $\mathrm{PCDD}_{25}$ (see Fig. 1).

Kruskall-Wallis and Kolmogorov-Smirnov tests showed that we can neglect the null hypothesis of similar dose distribution for at least half of the patients for $N<13$ (see Figs. 2 and 3, which show the number of patients with $p$ value larger than 0.01 for different values of $N$ ).

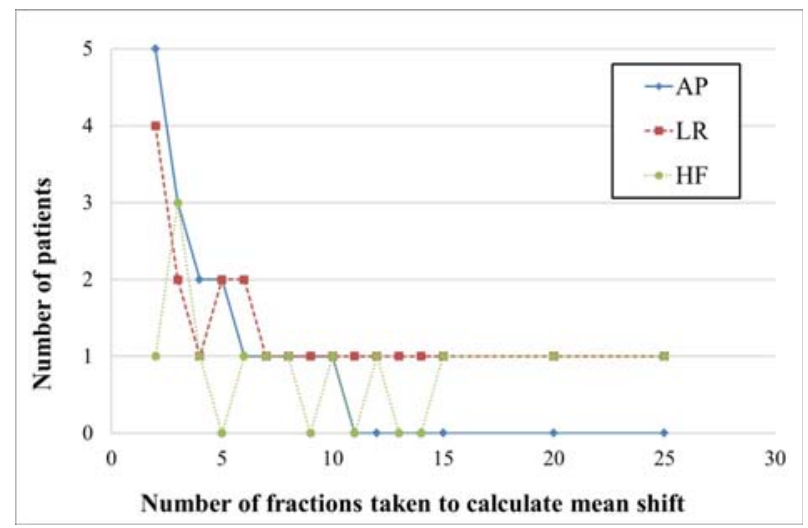

Fig. 1. Number of patients with gamma index $<1$ in less than $95 \%$ of points for AP, LR and HF profile, $\mathrm{PCDD}_{N}$ and CDD comparison. 


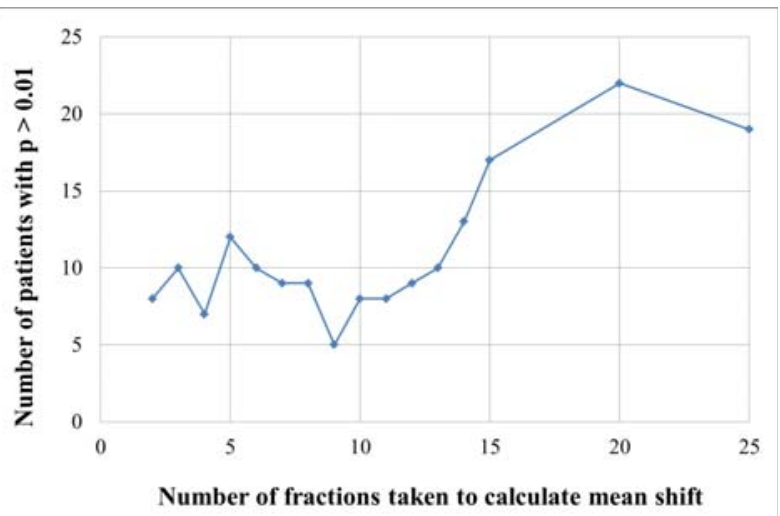

Fig. 2. Kruskall-Wallis test results for CTV. On $\mathrm{x}$ axis there is number of fractions taken to calculate mean shift and then $\operatorname{PCDD}_{N}$ (calculated by shifting isocenter in the TPS by a mean setup error value calculated from first $N$ fractions). On y axis there is number of patients with test result of $p$ value larger than 0.01 .

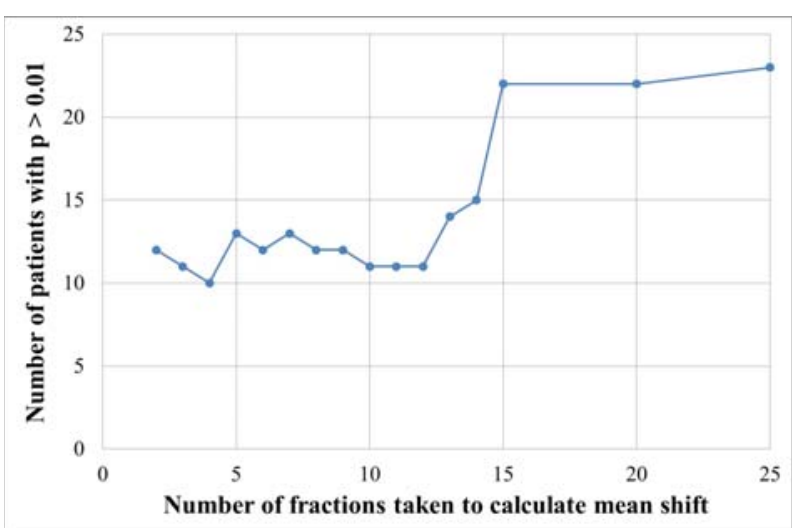

Fig. 3. Kolmogorov-Smirnov test results for CTV. On $x$ axis there is number of fractions taken to calculate mean shift and then $\operatorname{PCDD}_{N}$ (calculated by shifting isocenter in the TPS by a mean setup error value calculated from first $N$ fractions). On y axis there is number of patients with test result of $p$ value larger than 0.01 .

We used the Wilcoxon signed ranked test with statistical significance of 0.1 for two sets of data once for absolute mean dose values and once for percent dose values rounded to one-digit value. The result was that we have to decline the null hypothesis of no significant difference between mean values of CTV dose for $\mathrm{PCDD}_{20}$ and $\mathrm{PCDD}_{25}$ for the first method and for $\mathrm{PCDD}_{25}$ for the second method.

In almost all cases, the predicted minimum dose in CTV was lower than minimum dose for cumulated dose distribution. For all $\mathrm{PCDD}_{N}$ the predicted minimum dose in CTV was higher than $97 \%$ of dose for at least $75 \%$ of patients. Only single outsiders have predicted minimum dose less than $95 \%$. In Fig. 4, the difference between $\mathrm{CDD}$ and $\mathrm{PDD}_{N}$ in terms of the minimum dose to the CTV is presented. In Fig. 5 the predicted minimum dose to the CTV for different $N$ is given. The maximum dose for cumulative dose distribution never differs from the maximum dose for clinical treatment plan of more than $1 \%$.

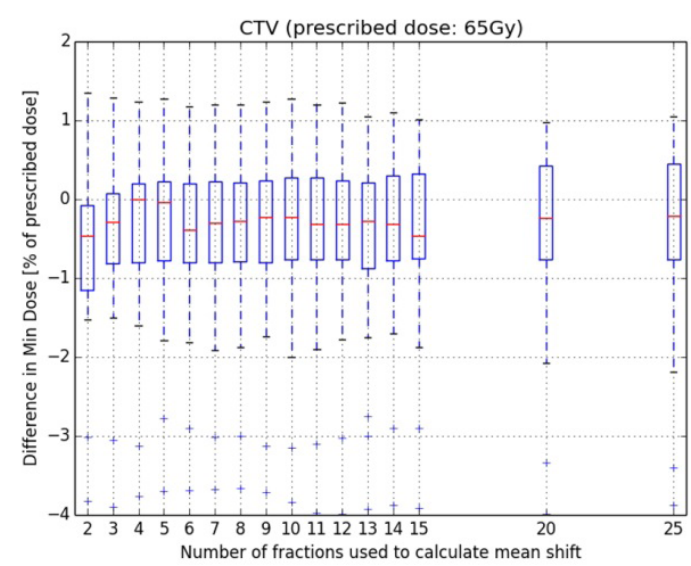

Fig. 4. Difference in Min Dose to CTV between $\operatorname{PCDD}_{N}$ (calculated by shifting isocenter in the TPS by a mean setup error value calculated from first $N$ fractions) and CDD. The line inside the box stands for median difference in Min Dose to CTV. Boxes are from 25th to 75 th percentile.

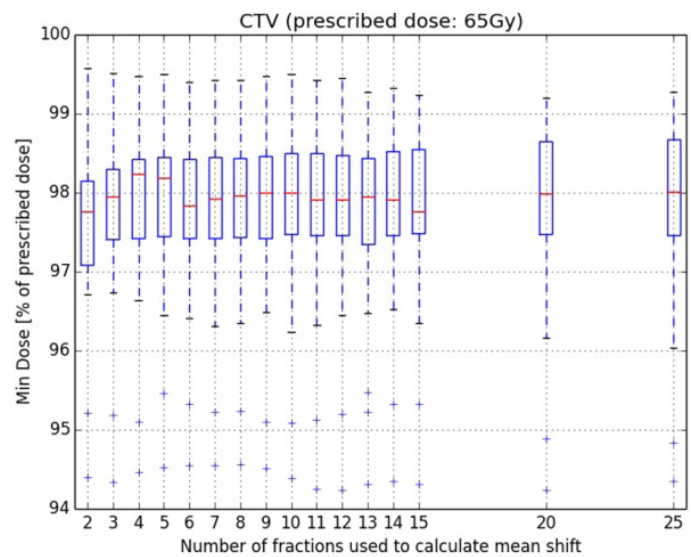

Fig. 5. Predicted Min Dose in CTV calculated for $\mathrm{PCDD}_{N}$ (calculated by shifting isocenter in the TPS by a mean setup error value calculated from first $N$ fractions). The line inside the box stands for percent value of Min Dose to CTV. Boxes are from 25 th to 75 th percentile.

\section{Discussion}

Our hypothesis was that it is possible to predict the final cumulated dose distribution after first few fractions. From our results, we can see that in fact after calculation of mean shift for seven first fractions, we can predict CDD fairly well. Also minimum dose to CTV is predicted properly.

Our statistical comparison of CTV dose distribution showed compatibility for half of patients (Kolmogorov-Smirnov test). However, paired $t$-test is usually used in similar studies, we think that nonparametric Wilcoxon signed ranked test is better suited for to test the hypothesis that the distribution in CTV for $\mathrm{PCDD}_{N}$ is the same as for $\mathrm{CDD}_{\text {all }}$ in terms of mean dose in CTV [12]. This is due to the fact that dose distribution in CTV is not normal. We can see that $\mathrm{PCDD}_{20}$ and $\mathrm{PCDD}_{25}$ disagreed our null hypothesis. To our surprise, the differences of mean doses between $\mathrm{PCDD}_{20}, \mathrm{PCDD}_{25}$ and CDD were statistically significant. However, they were very small, less than $0.2 \%$. Such small differences have negligible influence on treatment outcome. 
Our study was limited only to 3D-CRT technique, but we can guess that for other techniques such as intensity modulated radiation therapy (IMRT) and volumetric modulated arc therapy (VMAT) due to the higher dose gradient at the PTV border and higher low dose volume, the results might be similar as for 3D-CRT. Testing our method for other techniques will be a further step in our investigation.

It should be mentioned that in case of prostate its movement with respect to bony anatomy influences the cumulated dose distribution but the aim of this study was to test whether proposed method may be applied for targets for which internal movement is negligible. The results of this work may be applied for all these clinical situations were there the setup error is the main factor influencing on the dose distribution and if the dose distribution of setup errors is similar to the one obtained for prostate. We think that it may be applied for the brain patients. The individualization of the margin will be addressed in the next paper.

\section{Conclusion}

If we know setup errors for first seven fractions, the prediction of the cumulated dose distribution is quite robust. Based on the estimation of the cumulative dose distribution, the methods of margin individualization may be searched.

\section{References}

1. International Commission on Radiation Units and Measurements. (1999). Prescribing, recording and reporting photon beam therapy (Supplement to ICRU Report 50). Bethesda, Maryland: ICRU. (ICRU Report 62).

2. van Herk, M., Remeijer, P., Rasch, C., \& Lebesque, J. V. (2000). The probability of correct target dosage: Dose-population histograms for deriving treatment margins in radiotherapy. Int. J. Radiat. Oncol. Biol. Phys., 47(4), 1121-1135. DOI: 10.1016/S03603016(00)00518-6.
3. Stroom, J. C., de Boer, H. C. J., Huizinga, H., \& Visser, A. G. (1999). Inclusion of geometrical uncertainties in radiotherapy treatment planning by means of coverage probability. Int. J. Radiat. Oncol. Biol. Phys., 43(4), 905-919. DOI: 10.1016/S0360-3016(98)00468-4.

4. Rassiah-Szegedi, P., Wang, B., Szegedi, M., Tward, J., Zhao, H., Huang, Y. J., Sarkar, V., Shrieve, D., \& Salter, B. (2011). Individualized margins for prostate patients using a wireless localization and tracking system. J. Appl. Clin. Med. Phys., 12(3), 194-204. http://dx.doi.org/10.1120/jacmp.v12i3.3516.

5. Cheung, P., Sixel, K., Morton, G., Loblaw, D. A., Tirona, R., Pang, G., Choo, R., Szumacher, E., Deboer, G., \& Pignol, J. P. (2005). Individualized planning target volumes for intrafraction motion during hypofractionated intensity-modulated radiotherapy boost for prostate cancer. Int. J. Radiat. Oncol. Biol. Phys., 62(2), 418-425. DOI: 10.1016/j.ijrobp.2004.09.051.

6. Adamczyk, M., Piotrowski, T., Adamiak, E., \& Malicki, J. (2014). Dosimetric consequences of prostate-based couch shifts on the precision of dose delivery during simultaneous IMRT irradiation of the prostate, seminal vesicles and pelvic lymph nodes. Phys. Medica, 30(2), 228-233.

7. Piziorska, M., Kukołowicz, P., Zawadzka, A., Pilichowska, M., \& Peczkowski, P. (2012). Adaptive off-line protocol for prostate external radiotherapy with cone beam computer tomography. Strahlentherapie Onkol., 188(11), 1003-1009.

8. Depuydt, T., Van Esch, A., \& Huyskens, P. (2002). A quantitative evaluation of IMRT dose distributions: refinement and clinical assessment of the gamma evaluation. Radiother. Oncol., 62, 309-319. DOI: 10.1016/S0167-8140(01)00497-2.

9. Kruskal, W. H., \& Wallis, W. A. (1952). Use of ranks in one-criterion variance analysis. J. Am. Stat. Assoc., 47(260), 583-621. DOI: 10.1080/01621459.1952.10 483441 .

10. Smirnov, N. V. (1994). Approximate distribution laws for random variables, constructed from empirical data. Uspekhi Mat. Nauk, 10, 179-206. (in Russian).

11. Brahme, A. (1984). Dosimetric precision requirements in radiation therapy. Acta Radiol. Oncol. Radiat. Phys. Biol., 23(5), 379-391.

12. Lips, I., van der Heide, U., Kotte, A., van Vulpen, M., \& Bel, A. (2009). Effect of translational and rotational errors on complex dose distributions with off-line and on-line position verification. Int. J. Radiat. Oncol. Biol. Phys., 74(5), 1600-1608. DOI: 10.1016/j. ijrobp.2009.02.056. 